\title{
A classical Odderon in QCD at high energies
}

\author{
Sangyong Jeon \\ Physics Department, McGill University, Montreal, QC H3A-2T8, Canada \\ and RIKEN-BNL Research Center, Brookhaven National Laboratory, Upton, NY 11973 \\ Raju Venugopalan * \\ Physics Department, Brookhaven National Laboratory, Upton, NY 11973, USA.
}

November 15, 2018

\begin{abstract}
We show that the weight functional for color sources in the classical theory of the Color Glass Condensate includes a term which generates Odderon excitations. Remarkably, the classical origin of these excitations can be traced to the random walk of partons in the two dimensional space spanned by the $\mathrm{SU}(3)$ Casimirs. This term is naturally suppressed for a large nucleus at high energies.
\end{abstract}

\footnotetext{
*Present address: Institute for Theoretical Physics II, Univ. of Hamburg, Luruper Chaussee 49, 22607 Hamburg, Germany
} 


\section{Introduction}

Hadronic cross-sections have been successfully described for some time [1] in terms of non-perturbative t-channel exchanges known as Pomerons and Reggeons. In perturbative QCD (pQCD), the Pomeron arises from the color singlet exchange of two reggeized gluons in the t-channel [2]. This BFKL Pomeron, leads to cross-sections that grow rapidly as a power of the energy. Next-to-leading order corrections to the BFKL Pomeron [3], as well as unitarization and saturation corrections, are topics of active interest in high energy QCD [4].

The Pomeron, which corresponds to a C-even exchange in the t-channel, has a C-odd partner dubbed the Odderon [5]. In pQCD, it arises from the color singlet exchange of three reggeized gluons in the t-channel [6]. This BKP Odderon, in contrast to the BFKL Pomeron, has solutions that grow at most logarithmically as a function of the energy [7. A nice review of the Odderon can be found in Ref. 8].

Recently, Kovchegov et al. (KSW) [9], working in the dipole picture [10, 11], computed non-linear saturation corrections to the BKP Odderon equation in the limit of large number of colors $N_{c}$ and large atomic number $A$. These are analogous to the saturation correction derived previously [12] for the BFKL Pomeron. These saturation effects modify the BKP result, leading to an Odderon contribution to high energy amplitudes that decreases with energy.

Understanding the origins and role of the Odderon and its Pomeron partner, is essential for a consistent formulation of high energy QCD. One 
such attempt is the Reggeon effective field theory of Lipatov [13]. An alternative approach is the Color Glass Condensate [14] (CGC), where the degrees of freedom are parton fields at small x's of interest coupled to static parton sources at larger values of $\mathrm{x}$ [15]; their dynamical evolution with $\mathrm{x}$ is described by the JIMWLK Wilsonian renormalization group equations [16]. Understanding the correspondence between the two approaches will help clarify our understanding of QCD at high energies.

With this aim in mind, Hatta et al. [17] identified the dipole and baryon operators with Odderon quantum numbers in the CGC framework. The JIMWLK equations for the evolution of these operators are shown to be equivalent to the BKP equation for low parton densities. In the large $N_{c}$ and large $A$ mean field approximation, the KSW evolution equation for the Odderon dipole operator is recovered. The JIMWLK equations, in full generality, reveal a complex pattern of Pomeron-Odderon couplings under quantum evolution. Ref. 17] establishes a specific correspondence between the framework of Reggeon field theory and the Color Glass Condensate.

The authors of Ref. [17] also argue that Odderon excitations are absent in the McLerran-Venugopalan (MV) model [15], the classical effective theory (without quantum evolution) of the CGC. Their reasoning is correct for the original MV ansatz which assumed a Gaussian random distribution of the large $\mathrm{x}$ color sources in the CGC. However, we showed in a recent paper that the MV model for an $S U\left(N_{c}\right)$ gauge theory where $N_{c} \geq 3$ includes additional contributions to the distribution of sources [18. In this note, we will show that these contributions, sub-leading in the large $A$ limit, generate the Odderon. For SU(3), this term in the path integral of the MV model is 
proportional to the cubic Casimir operator. As in the small $\mathrm{x}$ limit, where (quantum) saturation suppresses Odderon excitations, (classical) saturation will be shown explicitly to suppress these as well.

This letter is organized as follows. We begin by summarizing briefly the MV model emphasizing the coarse graining assumption implicit in the model. We state recursion relations (derived previously in Ref. [18]) which describe the random walk in color space of $S U(3)$ quark color sources. We extend the discussion of Ref. [18 to explicitly write down the path integral for the SU(3) MV model. With this path integral, we compute the Odderon operators identified in Ref. [17] both in the weak field limit and to all orders in the classical field. In the weak field limit, for $A=1$, our results for these agree with Ref. [17].

\section{The MV path integral for a large nucleus and an $\mathrm{SU}(3)$ random walk}

The MV model is a simple model which captures several key features of QCD at high parton densities [15. The model can be generalized and the JIMWLK evolution equations we referred to previously arise from a proper accounting of quantum corrections in the Leading Logaritmic Approximation in $\mathrm{x}$ 16. The degrees of freedom are valence partons, which are random, static, light cone sources, and wee parton fields which couple coherently to valence sources all along the Lorentz contracted width of the nucleus. The kinematic condition for this coherence is $x \ll A^{-1 / 3}$. How many random sources the wee parton fields couple to depends on the typical transverse 
momentum of the wee parton ${ }^{1}$. A wee parton with momentum $p_{\perp}$ resolves in the transverse plane of the nucleus, an area $\left(\Delta x_{\perp}\right)^{2} \sim 1 / p_{\perp}^{2}$. The number of valence partons it interacts simultaneously with is then

$$
k \equiv k_{\left(\Delta x_{\perp}\right)^{2}}=\frac{N_{\text {valence }}}{\pi R^{2}}\left(\Delta x_{\perp}\right)^{2}
$$

which indeed is proportional to $A^{1 / 3}$ since $N_{\text {valence }}=3 \cdot A$ in QCD. This counting of color charges is only valid as long as $p_{\perp}>\Lambda_{\mathrm{QCD}}$.

The classical effective Lagrangean for the MV-model, formulated in the infinite momentum frame $\left(P^{+} \rightarrow \infty\right)$ and light cone gauge $\left(A^{+}=0\right)$ has the form,

$$
\mathcal{L}=S[A, \rho]+i W[\rho]
$$

Here $S[A, \rho]$ has two terms. The first is the usual QCD Field Strength tensor squared describing the dynamics of the wee parton gauge fields $A^{\mu}$. The second describes the coupling of these fields to the source color charge density $\rho$. These have been discussed previously [14 and will not concern us further here.

Our focus will be on the final term-corresponding to a Boltzmann-like weight $\exp (-W[\rho])$ in the generating functional for the effective action. It denotes the likelihood of different $\rho$ configurations. In the original MV model, the weight functional for a very large nucleus had the Gaussian form

$$
W[\rho]=\int d^{2} \mathbf{x} \frac{\rho^{a}(\mathbf{x}) \rho^{a}(\mathbf{x})}{2 \mu_{A}^{2}},
$$

\footnotetext{
${ }^{1}$ The wee parton is soft only in its longitudinal momentum-its transverse momentum may be large.
} 
where the average color charge squared per unit area per color degree of freedom, $\mu_{A}^{2}$ is simply determined to be [14, 18],

$$
\mu_{A}^{2}=\frac{g^{2} A}{2 \pi R^{2}} .
$$

It is of order $A^{1 / 3} \mathrm{fm}^{-2}$. For a large nucleus, this scale is the only dimensionful scale in Eq. 2. Thus $\alpha_{S}\left(\mu_{A}^{2}\right) \ll 1$, and all features of the theory can be computed in weak coupling.

In Ref. [18, it was shown explicitly that the assumption about the Gaussian distribution of charges for $k>>1$ is exact only for quark sources in an $\mathrm{SU}(2)$ gauge theory ${ }^{2}$. For $\mathrm{SU}(3)$ quarks, there is an additional contribution to Eq. 3. Since the argument has been stated at length previously in Ref. [18], we shall only outline key features below with emphasis on novel results.

The problem of interest can be formulated as follows. Given $k$ random $S U(3)$ quarks in a coarse grained cell of transverse area $\left(\Delta x_{\perp}\right)^{2}$, what is the distribution of color representations? This problem can be formulated straightforwardly as a recursion problem.

When one adds a quark to an $(m, n)$ representation of $\mathrm{SU}(3)$, the result is a sum of three irreducible representations ${ }^{3}$,

$$
(1,0) \times(m, n)=(m+1, n)+(m-1, n+1)+(m, n-1)
$$

\footnotetext{
${ }^{2}$ It is also true for gluon and quark-anti-quark sources in an $\mathrm{SU}(3)$ gauge theory. For a detailed discussion, see Ref. 18. This fact also explains why only the Gaussian term was obtained in the computation of Ref. [20], since there the nucleon was modeled as a quark-anti-quark dipole.

${ }^{3}$ Representations in $S U(3)$ can be expressed as $(m, n)$, where $m$ and $n$ are integers which label the upper and lower tensor indices respectively. A fundamental quark $\mathbf{3}$ state in $S U(3)$ is $(1,0)$ while an anti-quark in the $\overline{\mathbf{3}}$ state is $(0,1)$.
} 
One can deduce therefore that the multiplicity of a particular representation $N_{m, n}^{(k+1)}$ in the distribution of $k+1$ quarks (among all possible representations) is given by the recursion relation

$$
N_{m, n}^{(k+1)}=N_{m-1, n}^{(k)}+N_{m+1, n-1}^{(k)}+N_{m, n+1}^{(k)}
$$

for $m, n \geq 0$ with the boundary condition $N_{m,-1}^{(k)}=N_{-1, n}^{(k)}=0$ and the initial condition, $N_{0,0}^{(0)}=1$ else $N_{m, n}^{(0)}=0$.

As shown in Ref.[18], the multiplicity $N_{m, n}^{(k)}$ can be expressed in terms of the tri-nomial function $G_{k ; m, n}$ defined as

$$
G_{k: m, n}=\frac{k !}{\left(\frac{k+2 m+n}{3}\right) !\left(\frac{k-m+n}{3}\right) !\left(\frac{k-m-2 n}{3}\right) !} .
$$

One finds that

$$
\begin{aligned}
N_{m, n}^{(k)}= & G_{k: m, n}+G_{k: m+3, n}+G_{k: m, n+3} \\
& -G_{k: m+2, n-1}-G_{k: m-1, n+2}-G_{k: m+2, n+2}
\end{aligned}
$$

satisfies the recursion relation Eq. (6) with the stated initial and boundary conditions.

The two Casimirs for a given $S U(3) m, n$ state are the quadratic and cubic Casimirs [21,

$$
\begin{aligned}
D_{2}^{(m, n)} & =\frac{1}{3}\left(m^{2}+m n+n^{2}\right)+(m+n) \\
D_{3}^{(m, n)} & =\frac{1}{18}(m+2 n+3)(n+2 m+3)(m-n) .
\end{aligned}
$$

Note that while the former is symmetric in $m$ and $n$, the latter is antisymmetric under exchange of $m$ and $n$. For large $k$, one can use the Stirling 
formula to simplify Eq. [7] and further use Eq. 9] to obtain the very simple result:

$$
G_{k: m, n} \approx \frac{3^{\frac{3}{2}+k}}{2 k \pi} \exp \left(-3 D_{2}^{m, n} / k\right)\left(1+3 D_{3}^{m, n} / k^{2}\right)
$$

The multiplicity, defined in terms of the G's in Eq. 8, can be approximated in the large $k$ limit as

$$
\begin{aligned}
N_{m, n}^{(k)} & \approx 2 \partial_{m}^{3} G_{k: m, n}+2 \partial_{n}^{3} G_{k: m, n}-3 \partial_{m} \partial_{n}^{2} G_{k: m, n}-3 \partial_{n} \partial_{m}^{2} G_{k: m, n} \\
& \approx \frac{27 m n(m+n)}{k^{3}} \frac{3^{\frac{3}{2}+k}}{2 k \pi} \exp \left(-3 D_{2}^{m, n} / k\right)\left(1+3 D_{3}^{m, n} / k^{2}\right)
\end{aligned}
$$

While Eq. 10] was derived in Ref. [18, the final expression in Eq. 11] is new. The fact that the correction due to the cubic Casimir in Eq. 11] is identical to that in Eq. 10 is non-trivial and remarkable. From Eq. 9, one can deduce that the peak of the distribution is at $m=O(\sqrt{k})$ and $n=O(\sqrt{k})$. Correspondingly, the cubic Casimir introduces a correction of size $O(1 / \sqrt{k})$ for large $\mathrm{k}$.

The dimension of an $\mathrm{SU}(3)$ representation is

$$
d_{m n}=\frac{(m+1)(n+1)(m+n+2)}{2} \approx \frac{m n(m+n)}{2}
$$

The probability to find the system of $k$ quarks in an $(m, n)$ representation is therefore proportional to $d_{m n} N_{m, n}^{(k)}$. One can then define a normalized distribution ${ }^{4}$ in the sense that

$$
1=\frac{27 \sqrt{3}}{4 k^{4} \pi} \int_{0}^{\infty} d m d n m^{2} n^{2}(m+n) e^{-3 D_{2}^{m, n} / k}\left(1+3 D_{3}^{m, n} / k^{2}\right) .
$$

\footnotetext{
${ }^{4}$ One should not interpret the integrand as a probability density when $m$ and $n$ are large compared to $\sqrt{k}$. But this is academic since the probability to find such large $m$ 's and $n$ 's is exponentially suppressed.
} 
Since the cubic Casimir changes sign if $m$ and $n$ are exchanged, the correction term gives a vanishing contribution to the integral.

In Ref. [18, we proved that the right hand side of Eq. 13] can be rewritten exactly as an integral over classical color charges ${ }^{5}$,

$$
1 \approx\left(\frac{N_{c}}{k \pi}\right)^{4} \int d^{8} \mathbf{Q} e^{-N_{c} \mathbf{Q}^{2} / k+3 D_{3}(\mathbf{Q}) / k^{2}},
$$

where the cubic Casimir term in the integral is interpreted as a perturbation to the dominant quadratic Casimir term. Here $\mathbf{Q}=\left(Q_{1}, Q_{2}, \cdots, Q_{8}\right)$ is a classical color charge vector defined by $|\mathbf{Q}|=\sqrt{Q^{a} Q^{a}} \equiv \sqrt{D_{2}^{m, n}}$ and $Q_{1}, \cdots, Q_{8}$ are its eight components. The cubic Casimir, in terms of the color charges, is $D_{3}(Q)=d_{a b c} Q^{a} Q^{b} Q^{c}$, where $d_{a b c}$ is the symmetric tensor in the $S U(3)$ Lie algebra.

$Q^{a}$ is the classical color charge in a single coarse grained box. One can define a local classical color charge density $\rho^{a}$ in terms of $Q^{a}$ :

$$
Q_{a}=\frac{1}{g} \int_{\left(\Delta x_{\perp}\right)^{2}} d^{2} \mathbf{x} \rho_{a}(\mathbf{x}) \approx \frac{1}{g}\left(\Delta x_{\perp}\right)^{2} \rho_{a}(\mathbf{x}) .
$$

With this definition, and $k$ defined as in Eq. 1, one can write down a path integral for the distribution of the color charges as

$$
1 \approx \int[d \rho] \exp \left(-\int_{A} d^{2} \mathbf{x}\left[\frac{\rho_{a}(\mathbf{x}) \rho_{a}(\mathbf{x})}{2 \mu_{A}^{2}}-\frac{d_{a b c} \rho^{a}(\mathbf{x}) \rho^{b}(\mathbf{x}) \rho^{c}(\mathbf{x})}{\kappa_{A}}\right]\right) .
$$

where $\mu_{A}^{2}=g^{2} A / 2 \pi R_{A}^{2}$ was defined in Eq. 4. For the weight of the cubic Casimir, we obtain,

$$
\kappa_{A}=\frac{g^{3} A^{2} N_{c}}{\pi^{2} R^{4}} .
$$

As we will now see, the cubic term in Eq. 16 is precisely the term that gives rise to the Odderon.

\footnotetext{
${ }^{5}$ Our derivation did not include the cubic Casimir but that does not change anything since that term integrates to zero.
} 


\section{Odderon in the classical effective theory}

In Ref. [17, the authors identified the Odderon exchange operators in two situations, a) a dipole scattering off the CGC, and b) a three quark baryon system scattering off the CGC. We first consider the former where the C-odd "dipole odderon operator" is defined to be

$$
\mathcal{O}(\mathbf{x}, \mathbf{y})=\frac{1}{2 i N_{c}} \operatorname{Tr}\left(V_{x}^{\dagger} V_{y}-V_{y}^{\dagger} V_{x}\right)
$$

where

$$
V_{x}^{\dagger} \equiv V_{-\infty, \infty}^{\dagger}(\mathbf{x})=P \exp \left(i g \int_{-\infty}^{\infty} d x^{-} \alpha^{a}\left(x^{-}, \mathbf{x}\right) t^{a}\right)
$$

and $V_{-\infty, \infty}^{\dagger}=V_{\infty,-\infty}$. Here $t^{a}$ is a generator in the fundamental representation of $S U(3)$ and $\alpha^{a}$ is the CGC gauge field. In the weak field limit, one can expand out the path ordered exponentials-the lowest order contributing to the Odderon is of cubic order in $\alpha$ [17]:

$$
\mathcal{O}(\mathbf{x}, \mathbf{y}) \approx \frac{-g^{3}}{24 N_{c}} d^{a b c}\left(\alpha_{x}^{a}-\alpha_{y}^{a}\right)\left(\alpha_{x}^{b}-\alpha_{y}^{b}\right)\left(\alpha_{x}^{c}-\alpha_{y}^{c}\right)
$$

Now, from the classical equations of motion for gauge fields in the CGC, the gauge field can be expressed in term of the classical color charge density $\rho^{a}$ in Lorentz gauge as

$$
\alpha_{x}^{a}=\frac{1}{4 \pi} \int d^{2} \mathbf{z} \ln \left(\frac{1}{(\mathbf{x}-\mathbf{z})^{2} \Lambda^{2}}\right) \rho^{a}(\mathbf{z}),
$$

where $\Lambda$ is an infra-red cut-off which will not appear in the final result. From this relation therefore, one expects $\mathcal{O} \propto d^{a b c} \rho^{a} \rho^{b} \rho^{c}$. In the original MV ansatz, the expectation value of this Odderon operator would be zero. 
However, from Eq. 16. we now know that there is indeed a contribution (albeit suppressed) in the source term of the MV model which has the color structure of the cubic Casimir operator $d^{a b c} \rho^{a} \rho^{b} \rho^{c}$. One can therefore compute the initial condition for quantum evolution of the CGC directly from the MV model.

To evaluate $\langle\mathcal{O}\rangle$, with the measure in Eq. [16, one needs to compute the integral

$$
\begin{aligned}
\mathcal{I} & =\frac{d_{a b c} d_{\bar{a} \bar{b} \bar{c}}}{Z_{0} \kappa_{A}} \int[d \rho] e^{-\int d^{2} \mathbf{x} \rho^{2} / 2 \mu_{A}^{2}} \\
& \times \int d^{2} \mathbf{y} \rho^{a}(\mathbf{y}) \rho^{b}(\mathbf{y}) \rho^{c}(\mathbf{y}) \rho^{\bar{a}}(\mathbf{u}) \rho^{\bar{b}}(\mathbf{v}) \rho^{\bar{c}}(\mathbf{w}) .
\end{aligned}
$$

As prescribed previously, we have expanded out the cubic piece in Eq. 16 here. $Z_{0}$ corresponds to this measure. $\mathcal{I}$ can be easily computed using Wick's theorem. Ignoring disconnected pieces for the moment, one obtains,

$$
\mathcal{I}=6 \frac{d_{a b c} d_{\bar{b} \bar{c} \bar{c}}}{Z_{0} \kappa_{A}} \int d^{2} \mathbf{y}\left\langle\rho^{a}(\mathbf{y}) \rho^{\bar{a}}(\mathbf{u})\right\rangle\left\langle\rho^{b}(\mathbf{y}) \rho^{\bar{b}}(\mathbf{v})\right\rangle\left\langle\rho^{c}(\mathbf{y}) \rho^{\bar{c}}(\mathbf{w})\right\rangle
$$

where we used the fact that $d_{a b c}$ is a totally symmetric tensor. In the MV model, the 2-point correlator is simply

$$
\left\langle\rho^{a}(\mathbf{x}) \rho^{b}(\mathbf{y})\right\rangle=\mu_{A}^{2} \delta^{a b} \delta^{(2)}(\mathbf{x}-\mathbf{y}) .
$$

Using Eqs. 21]24 to compute the expectation value of $\mathcal{O}$, one obtains,

$$
\begin{aligned}
\langle\mathcal{O}(\mathbf{x}, \mathbf{y})\rangle & =\frac{-g^{3} \mu_{A}^{6}}{4 N_{c} \kappa_{A}} d^{a b c} d_{a b c} \int d^{2} \mathbf{u}(G(\mathbf{x}-\mathbf{u})-G(\mathbf{y}-\mathbf{u}))^{3} \\
& =\alpha_{S}^{3} \frac{\left(N_{c}^{2}-4\right)\left(N_{c}^{2}-1\right)}{4 \pi r_{0}^{2} N_{c}^{3}} A^{1 / 3} \int d^{2} \mathbf{u} \ln ^{3} \frac{|\mathbf{x}-\mathbf{u}|}{|\mathbf{y}-\mathbf{u}|},
\end{aligned}
$$


where we defined $G(\mathbf{x}-\mathbf{y})=\ln \left(\frac{1}{(\mathbf{x}-\mathbf{y})^{2} \Lambda^{2}}\right) / 4 \pi$. Also, note that $\mu_{A}^{2}$ and $\kappa_{A}$ were defined in Eq. 4 and Eq. 17 respectively, $r_{0}=1.12 \mathrm{fm}$ and we used the identity $d_{a b c} d^{a b c}=\left(N_{c}^{2}-1\right)\left(N_{c}^{2}-4\right) / N_{c}$.

In performing this computation, we ignored the disconnected pieces coming from the Wick expansion of Eq.(22). These pieces vanish because they involve a summation with two contracted indices of $d_{a b c}$.

We now turn to the Odderon operator corresponding to a three quark baryon state scattering off the CGC. In that case, the relevant operator is [23, 17]

$$
\mathcal{B}(\mathbf{x}, \mathbf{y}, \mathbf{z})=\frac{1}{3 ! 2 i}\left(\epsilon^{i j k} \epsilon^{l m n} V_{i l}^{\dagger}(\mathbf{x}) V_{j m}^{\dagger}(\mathbf{y}) V_{k n}^{\dagger}(\mathbf{z})-\text { h.c. }\right) .
$$

where $V_{x}^{\dagger}$ is defined in Eq. 19. Again, expanding out to lowest non-trivial order in $\alpha$, the expression for $\mathrm{B}(\mathbf{x}, \mathbf{y}, \mathbf{z})$ in the weak field limit is

$$
\approx \frac{g^{3}}{144} d^{a b c}\left\{\left(\alpha_{x}^{a}+\alpha_{y}^{a}-2 \alpha_{z}^{a}\right)\left(\alpha_{y}^{b}+\alpha_{z}^{b}-2 \alpha_{x}^{b}\right)\left(\alpha_{z}^{c}+\alpha_{x}^{c}-2 \alpha_{y}^{c}\right)\right\}
$$

Replacing $\alpha \rightarrow \rho$ using Eq. 21, using Wick's theorem and computing the average over color sources using Eq. 24, one obtains,

$$
\begin{aligned}
\langle\mathcal{B}\rangle & =\frac{g^{3} \mu_{A}^{6}}{144} d^{a b c} d_{a b c} \frac{6}{\kappa_{A}} \int d^{2} \mathbf{u}(G(\mathbf{x}-\mathbf{u})+G(\mathbf{y}-\mathbf{u})-2 G(\mathbf{z}-\mathbf{u})) \\
& \times(G(\mathbf{z}-\mathbf{u})+G(\mathbf{x}-\mathbf{u})-2 G(\mathbf{y}-\mathbf{u})) \\
& \times(G(\mathbf{y}-\mathbf{u})+G(\mathbf{z}-\mathbf{u})-2 G(\mathbf{x}-\mathbf{u})) \\
& =\frac{\alpha_{S}^{3}}{24 \pi r_{0}^{2}} \frac{\left(N_{c}^{2}-4\right)\left(N_{c}^{2}-1\right)}{N_{c}^{2}} A^{1 / 3} \int d^{2} \mathbf{u} \ln \frac{|\mathbf{x}-\mathbf{u}||\mathbf{y}-\mathbf{u}|}{|\mathbf{z}-\mathbf{u}|^{2}} \\
& \times \ln \frac{|\mathbf{y}-\mathbf{u}||\mathbf{z}-\mathbf{u}|}{|\mathbf{x}-\mathbf{u}|^{2}} \ln \frac{|\mathbf{z}-\mathbf{u}||\mathbf{x}-\mathbf{u}|}{|\mathbf{y}-\mathbf{u}|^{2}}
\end{aligned}
$$

For $N_{c}=3$ and $A=1$, both Eq. 25 and Eq. 28 can be shown to agree exactly with Eqs. 5.5 and 6.2 respectively in Ref. 17. To compare, we need 
to assume the nucleon is dilute and the fact that the result of Ref. [17] is for a single quark (namely divide our result by 3). In Eq. 25 and Eq. 28, the factor of $A^{1 / 3}$ accounts for the possibility that the Odderon couples to $A^{1 / 3}$ nucleons in the nucleus.

\section{Result to all orders in the gluon density}

We shall now explicitly evaluate the expectation value of the dipole odderon operator in Eq. [18 to all orders in $g^{2} \mu_{A}^{2} / k_{\perp}^{2}$. In performing this computation, we have to take into account the path ordering of the gauge field in the $x^{-}$ direction-see Eq. 19]. The expectation value of $\mathcal{O}(\mathbf{x}, \mathbf{y})$ is given by

$$
\begin{aligned}
\langle\mathcal{O}\rangle & =\frac{d_{a b c}}{2 i N_{c} \kappa_{A}} \int d u^{-} \int d^{2} \mathbf{u}\left\langle\rho_{a}\left(u^{-}, \mathbf{u}\right) \rho_{b}\left(u^{-}, \mathbf{u}\right) \rho_{b}\left(u^{-}, \mathbf{u}\right)\right. \\
& \left.\times \operatorname{Tr}\left(V(\mathbf{x})^{\dagger} V(\mathbf{y})-V(\mathbf{y})^{\dagger} V(\mathbf{x})\right)\right\rangle
\end{aligned}
$$

where the average is now done with the "smeared" Gaussian weight

$$
W[\rho]=\exp \left(-\int d z^{-} \int d^{2} \mathbf{z} \frac{\rho_{a}\left(z^{-}, \mathbf{z}\right) \rho_{a}\left(z^{-}, \mathbf{z}\right)}{2 \mu^{2}\left(z^{-}\right)}\right)
$$

Note that $\left\langle\rho^{a}\left(x^{-}, \mathbf{x}\right) \rho^{b}\left(y^{-}, \mathbf{y}\right)\right\rangle=\mu^{2}\left(x^{-}\right) \delta^{a b} \delta\left(x^{-}-y^{-}\right) \delta^{(2)}(\mathbf{x}-\mathbf{y})$. To evaluate $\langle\mathcal{O}\rangle$, we use the following identity,

$$
d_{a b c} \frac{\delta}{\delta \rho_{a}(u)} \frac{\delta}{\delta \rho_{b}(u)} \frac{\delta}{\delta \rho_{c}(u)} W[\rho]=-\frac{d_{a b c}}{\mu^{6}\left(u^{-}\right)} \rho_{a}(u) \rho_{b}(u) \rho_{c}(u) W[\rho]
$$

using the fact that $W$ is a Gaussian and $d_{a a b}$, summed over $a$ is zero. Re-

placing the LHS of this identity, which appears in Eq. 29, with the RHS and integrating by parts, we can write $\mathcal{O}$ as the sum of two terms, where the first is

$$
\begin{aligned}
\langle\mathcal{O}\rangle_{1} & =\frac{d_{a b c}}{2 i N_{c} \kappa_{A}} \int d u^{-} \mu^{6}\left(u^{-}\right) \int d^{2} \mathbf{u}\left\langle\frac{\delta}{\delta \rho_{a}(u)} \frac{\delta}{\delta \rho_{b}(u)} \frac{\delta}{\delta \rho_{c}(u)}\right. \\
& \left.\times \operatorname{Tr}\left(V_{-\infty, \infty}(\mathbf{y}) V_{\infty,-\infty}(\mathbf{x})\right)\right\rangle .
\end{aligned}
$$


The second term $\langle\mathcal{O}\rangle_{2}$ is obtained by changing the overall sign in $\langle\mathcal{O}\rangle_{1}$ and exchanging $\mathbf{x}$ and $\mathbf{y}$. After some algebra ${ }^{6}$,

$$
\begin{aligned}
\langle\mathcal{O}\rangle_{1} & =\frac{g^{3}\left(N_{c}^{2}-4\right)}{2 i N_{c}^{2} \kappa_{A}} \int d u^{-} \mu^{6}\left(u^{-}\right) \int d^{2} \mathbf{u}\left[\frac{\left(N_{c}^{2}-1\right) i}{4 N_{c}}\left(G^{3}(\mathbf{x}-\mathbf{u})-G^{3}(\mathbf{y}-\mathbf{u})\right)\right. \\
& \left.\times \mathcal{I}_{1}^{\infty,-\infty}+\frac{3 i}{2}\left(G^{2}(\mathbf{y}-\mathbf{u}) G(\mathbf{x}-\mathbf{u})-G(\mathbf{y}-\mathbf{u}) G^{2}(\mathbf{x}-\mathbf{u})\right) \cdot \mathcal{I}_{2}^{\infty,-\infty}\right](33)
\end{aligned}
$$

where $\mathcal{I}_{1}^{\infty,-\infty}=\left\langle V_{-\infty, \infty}(\mathbf{y}) V_{\infty,-\infty}(\mathbf{x})\right\rangle$ and

$\mathcal{I}_{2}^{\infty,-\infty}=\left\langle t_{a} V_{u^{-}, \infty}(\mathbf{y}) V_{\infty, u^{-}}(\mathbf{x}) t_{a} V_{u^{-},-\infty}(\mathbf{x}) V_{-\infty, u^{-}}(\mathbf{y})\right\rangle \cdot \mathcal{I}_{2}$ can be simplified ${ }^{7}$

using the locality of the distributions in $x^{-}$. Further, since [24, 25]

$$
\mathcal{I}_{1}^{v^{-}, u^{-}}=\exp \left(-\frac{g^{2} t_{a} t_{a}}{2} \int_{u^{-}}^{v^{-}} d z^{-} \mu^{2}\left(z^{-}\right) \int d^{2} \mathbf{z}(G(\mathbf{x}-\mathbf{z})-G(\mathbf{y}-\mathbf{z}))^{2}\right)
$$

and $t_{a} t_{a} \propto \mathbf{1}_{3 \times 3}$, it commutes with all $t_{a}$, and the two sets of $\left\langle V^{\dagger} V\right\rangle$ in the second term of Eq. 33 can be combined to form $\mathcal{I}_{1}^{\infty,-\infty}$. Substituting these results in Eq. 33, and combining it with $\left\langle\mathcal{O}_{2}\right\rangle$ (obtained by exchanging $\mathbf{x}$ and y), we have,

$$
\begin{aligned}
& \langle\mathcal{O}\rangle=\alpha_{S}^{3} \frac{\left(N_{c}^{2}-4\right)\left(N_{c}^{2}-1\right)}{4 \pi r_{0}^{2} N_{c}^{4}} A^{1 / 3} \int d^{2} \mathbf{u} \ln ^{3} \frac{|\mathbf{x}-\mathbf{u}|}{|\mathbf{y}-\mathbf{u}|}, \\
& \times \operatorname{Tr} \exp \left(-\frac{g^{2} \mu_{A}^{2} t_{a} t_{a}}{2} \int d^{2} \mathbf{z}(G(\mathbf{x}-\mathbf{z})-G(\mathbf{y}-\mathbf{z}))^{2}\right) .
\end{aligned}
$$

We have replaced here $\int_{-\infty}^{\infty} d z^{-} \mu^{2}\left(z^{-}\right)=\mu_{A}^{2}$. Note that $\operatorname{Tr} \exp ()=N_{c}-$ $\left(\left(N_{c}^{2}-1\right) / 4 \cdots\right.$, so the first term in the expansion is identical to the weak

${ }^{6}$ We have used the identities $d_{a b c} t_{a} t_{b}=\frac{1}{2} \frac{N_{c}^{2}-4}{N_{c}} t_{c}$ and $d_{a b c} t_{a} t_{b} t_{c}=\frac{\left(N_{c}^{2}-4\right)\left(N_{c}^{2}-1\right)}{4 N_{c}^{2}} \mathbf{1}$ where 1 is the $3 \times 3$ identity matrix. We have also used the cyclic property of the trace.

${ }^{7}$ The local structure in $x^{-}$(see the expression after Eq. 130) ensures that only adjacent Wilson lines can contracted [25]. This simplifies $\mathcal{I}_{2}^{\infty,-\infty}$ to read

$$
\mathcal{I}_{2}=t_{a}\left\langle V_{u^{-}, \infty}(\mathbf{y}) V_{\infty, u^{-}}(\mathbf{x})\right\rangle t_{a}\left\langle V_{u^{-},-\infty}(\mathbf{x}) V_{-\infty, u^{-}}(\mathbf{y})\right\rangle
$$


field result in Eq. 25. As discussed previously, the argument of the exponential here can be expressed in terms of the saturation scale $Q_{s, A}^{2}$ of a large nucleus [24, 25, 14]. Our expression in Eq. 34]agrees with the initial condition (modulo geometrical factors) for Odderon evolution suggested in Ref. [9] in a diagrammatic approach. In the large A limit, when the saturation scale is large, Eq. 34 tells us that the Odderon contribution will be suppressed. Of course, there is a further suppression that comes in from quantum evolution [17].

We shall now outline the computation of the Baryon Odderon operator to all orders in the classical color field. The full computation, while perfectly feasible and straightforward, is extremely tedious for reasons which will become clear. This will be left for a later date. We begin with an alternative expression to that in Eq. 26, also given in Ref. [17], which will suit us better:

$$
\begin{aligned}
\mathcal{B}(x, y, z) & =\frac{1}{3 ! 2 i}\left[\operatorname{Tr}\left(V_{x}^{\dagger} V_{z}\right) \operatorname{Tr}\left(V_{y}^{\dagger} V_{z}\right)-\operatorname{Tr}\left(V_{x}^{\dagger} V_{z} V_{y}^{\dagger} V_{z}\right)-\text { h.c. }\right] \\
& \equiv \mathcal{K}_{1}-\mathcal{K}_{2}-\text { h.c. }
\end{aligned}
$$

To compute the expectation value of this object, we follow the same procedure as that for the dipole Odderon operator. Consider for example the expectation value of the first term. Using the identity in Eq. 31, we have,

$$
\begin{aligned}
\langle\mathcal{K}\rangle_{1}= & \frac{d_{a b c}}{12 i \kappa_{A}} \int d u^{-} \int d^{2} \mathbf{u}\left\langle\frac{\partial}{\partial \rho_{a}(u)} \frac{\partial}{\partial \rho_{b}(u)} \frac{\partial}{\partial \rho_{c}(u)}\right. \\
& \left.\left(V_{-\infty, \infty}(\mathbf{x}) V_{\infty,-\infty}(\mathbf{z})\right)\left(V_{-\infty, \infty}(\mathbf{y}) V_{\infty,-\infty}(\mathbf{z})\right)\right\rangle .
\end{aligned}
$$

One has an analogous expression for $\left\langle\mathcal{K}_{2}\right\rangle$. Unlike Eq. 32 where one had to manipulate 8 terms (2 from each differentiation), one now has $4^{3}=64$ terms from $\left\langle\mathcal{K}_{1}\right\rangle$ and an equal number from $\left\langle\mathcal{K}_{2}\right\rangle$ to make a total of 128 terms! These 
can be combined using Fierz identities eventually into Gaussian correlators of 2-point, 3-point and 4-point Wilson lines. A general technique to compute these was discussed in Appendix A of Ref. [26.

\section{Summary}

We showed in this note that, for SU(3) quark sources, the MV model includes a term proportional to the cubic Casimir in the path integral. We obtained in Eq. [16] a quantitative expression for this correction. Remarkably, this term derived from a random walk analysis of classical color charges is responsible for generating Odderon excitations in the classical effective theory of the Color Glass Condensate. Our results for the expectation values of the dipole Odderon operator and the Baryon Odderon operator in the CGC background field agree exactly with those of Ref. [17] in the weak field and $A=1$ limit. In addition, we computed the expectation value of the dipole Odderon operator to all orders in the classical field and showed that the result agreed with Ref. [9]. We outlined the computation of the Baryon Odderon operator-the detailed computation will be left for a future study. Our results suggest that Odderon excitations, while quantitatively suppressed, arise naturally in a consistent mathematical framework and are therefore relevant for a systematic study of high energy QCD.

\section{Acknowledgments}

One of us (RV) would like to thank Jochen Bartels, Larry McLerran and Yuri Kovchegov for very useful discussions. We would like to thank Edmond Iancu for reading the manuscript. RV's research is supported in part by DOE 
Contract No. DE-AC02-98CH10886 and by a grant from the Alexander von Humboldt foundation. SJ is supported in part by the Natural Sciences and Engineering Research Council of Canada and by le Fonds Nature et Technologies of Québec. He also thanks RIKEN BNL Center and U.S. Department of Energy [DE-AC02-98CH10886] for providing facilities essential for the completion of this work.

\section{References}

[1] A. Donnachie and P. V. Landshoff, Phys. Lett. B 296, 227 (1992).

[2] E. A. Kuraev, L. N. Lipatov and V. S. Fadin, Sov. Phys. JETP 45, 199 (1977) [Zh. Eksp. Teor. Fiz. 72, 377 (1977)]; I. I. Balitsky and L. N. Lipatov, Sov. J. Nucl. Phys. 28, 822 (1978) [Yad. Fiz. 28, 1597 (1978)].

[3] V. S. Fadin and L. N. Lipatov, Phys. Lett. B 429, 127 (1998); M. Ciafaloni and G. Camici, Phys. Lett. B 430, 349 (1998).

[4] A. H. Mueller, arXiv:hep-ph/9911289 L. McLerran, Acta Phys. Polon. B 34, 5783 (2003); N. Armesto, Acta Phys. Polon. B 35, 213 (2004);

A. M. Stasto, arXiv:hep-ph/0412084.

[5] L. Lukaszuk and B. Nicolescu, Lett. Nuovo Cim. 8, 405 (1973).

[6] J. Bartels, Nucl. Phys. B 175 (1980) 365; J. Kwiecinski and M. Praszalowicz, Phys. Lett. B 94, (1980) 413.

[7] J. Bartels, L. N. Lipatov and G. P. Vacca, Phys. Lett. B 477, (2000) 178; R. A. Janik and J. Wosiek, Phys. Rev. Lett. 82, (1999) 1092. 
[8] C. Ewerz, hep-ph/0306137.

[9] Y. V. Kovchegov, L. Szymanowski and S. Wallon, Phys. Lett. B 586, 267 (2004).

[10] A. H. Mueller, Nucl. Phys. B 415, 373 (1994).

[11] N. N. Nikolaev and B. G. Zakharov, Z. Phys. C49, (1991) 607.

[12] I. Balitsky, Nucl. Phys. B 463, 99 (1996); Yu.V. Kovchegov, Phys. Rev. D 61, 074018 (2000).

[13] L. N. Lipatov, Phys. Rept. 286, 131 (1997); ibid., 320, 249 (1999).

[14] E. Iancu and R. Venugopalan, arXiv:hep-ph/0303204.

[15] L. D. McLerran and R. Venugopalan, Phys. Rev. D 49, 2233 (1994); ibid., 3352, (1994); ibid., 50, 2225 (1994).

[16] J. Jalilian-Marian, A. Kovner, A. Leonidov, H. Weigert, Nucl. Phys. B 504, 415 (1997); J. Jalilian-Marian, A. Kovner, A. Leonidov, H. Weigert, Phys. Rev. D 59, 014014 (1999); A. Kovner, G. Milhano, H. Weigert, Phys. Rev. D 62, 114005 (2000); E. Iancu, A. Leonidov, L.D. McLerran, Nucl. Phys. A 692, 583 (2001); E. Iancu, A. Leonidov, L.D. McLerran, Phys. Lett. B 510, 133 (2001); E. Ferreiro, E. Iancu, A. Leonidov, L.D. McLerran, Nucl. Phys. A 703, 489 (2002).

[17] Y. Hatta, E. Iancu, K. Itakura and L. McLerran, arXiv:hep-ph/0501171.

[18] S. Jeon and R. Venugopalan, Phys. Rev. D 70, 105012 (2004). 
[19] J. Jalilian-Marian, A. Kovner, L. D. McLerran and H. Weigert, Phys. Rev. D 55, 5414 (1997).

[20] Y. V. Kovchegov, Phys. Rev. D 54, 5463 (1996); ibid., 55, 5445 (1997).

[21] J.A.de Azcarraga and A.J Macfarlane, J. Math. Phys. 42, 419 (2001).

[22] J. C. Collins, "An Introduction To Renormalization, The Renormalization Group, And The Operator Product Expansion," Cambridge Univ. Press ( 1984) 380p.

[23] H. G. Dosch, E. Ferreira and A. Kramer, Phys. Rev. D 50, (1994) 1992;

H. G. Dosch, C. Ewerz and V. Schatz, Eur. Phys. J. C 24, 561 (2002).

[24] L. D. McLerran and R. Venugopalan, Phys. Rev. D 59, 094002 (1999);

R. Venugopalan, Acta Phys. Polon. B 30, 3731 (1999).

[25] F. Gelis and A. Peshier, Nucl. Phys. A 697, 879 (2002).

[26] J. P. Blaizot, F. Gelis and R. Venugopalan, Nucl. Phys. A 743, 57 (2004). 